die nom Zeituntersehied des Einsatzes herrühren, neben solchen auftreten, die won der PhasenZeit-Differenz stammen. Für die ersteren wird vermutlich eine geringere Intensitätsempfindlichkeit vorhanden sein als für die letzteren.

$\mathrm{E}_{\mathrm{S}}$ wăre sehr erwünscht, wenn unsere Versuche von anderer Seite wiederholt, erweitert und ihre Resultate bestätigt werden könnten. Desgleichen wäre die Frage der Halbkreisbestimmung durch evtl. Nitwirkung eines nicht durch den normalen Weg zum inneren Ohr gelangenden Schalles und die Frage der Reizschwelle für Intensitätsunterschiede abhängig von der Frequenz eingehender zu prüfen.

Auf dem Gebiete der Lokalisation von Schaliquellen ist bereits eine sehr arsgedehnte litera$\left.t u x^{3}\right)$ entstanden. Ich habe aber nicht den Eindruck, dab auber einer sehr fleißigen Zusammenstellung von allem möglichen Beobachtungsmaterial viel Grundsätzliches geleistet worden ist. Tch würde mich freuen, durch meine Ausführungen Anregung zum Arbeiten und Erforschen der grundlegenden Gesutze gegeben zu haben, deren Kenntnis sowohl fiis den Physiologen und Phy siker, wie anch für den praktischen Ingenienr von grober Bedeutung sind.

\section{Die Entstehung des Torfes und der Kohle.}

Von Hans Höfer $v$. Heimhalt, Wien.

Zweifelsohne hat die Studie von $F$. Fischer und $H$. Schrader: ,Entstehung und chemische Struktur der Kohle"is) auBerordentlich anregend gewirkt, wie dies auch aus den darauf erfolgten Widersprüchen geschlossen werden kann. Bezüglich des Trsprungmaterials der Kohle bildeten sich zwei Lager: "Hie Zellulose" — "Hie Lignin", während eine kleine Gruppe, zu der J. Marcusson gehört, Kohle aus Zellulose und Lignin ableitet; ja selbst die eingangs genannten Schöpfer der Ligninhypothese, welche die Zellulose schon zu Beginn der Verwandlung der Holzsubstanz verschwinden, d. h. in $\mathrm{CO}_{2}, \mathrm{CH}_{4}, \mathrm{H}_{2} \mathrm{O}$ und einige im Grundwasser lösliche Säuren verwandeln lassen, geben schließlioh (S. 31) zu: ,Jede Mitbeteiligung der Zellulose an der Kohlebildung wollen und können wir nicht bestreiten. Auf keinen Fall aber spielt sie die ausschlaggebende Polle, die man ihr bisher zugeschrieben hat." Viel allgemeiner folgerte schon viel früher $B$ er gius aus seinen klassischen Versuchen: „Die Ahnlichkeit der Zellulosekohle mit der aus Torf hergestellten ist ein Beweis dafür, daB das Ausgangsmaterial. solange es nur pflanzlichen Ur

3) Siehe Anmerkung 1 aut Seite 107.

1) Brennstoff-Chemie, 2. Bd., 1921, Verlag W. Giradet in Essen. - F. Fischer: Die Naturwissenschaften, Heft 47,1921

9) Die Anwendung hoher Drucke bei chemischen Vorgingen und eine Nachbildung des Entstehungsprozesses der Steinkohle, S. 50, Halle 1913. sprungs ist, loinen wesentlichen EinfluB auf die entstandenen Kohlen gehabt hat."

Ich sprach mich im Briefwechsel mit Kohlenchemikern sofort nach dem Erscheinen der Studie Fischer-Schraders für den Dualismus aus; es drängt mich die Frage der Entstehung der Kohlen als Grologe zu besprechen, um so mehr, da unsere Lehrbücher der allgemeinen Geologie sich meist begnügen, im Kohlebildungsprozel nur einen Abbau von $\mathrm{O}, \mathrm{H}$ und $\mathrm{N}$ bei relativer Anreicherung des $\mathrm{O}$ zu sehen, also das Sinnfällige der Analysen. Die jüngsten Spezialwerke über "Kohle" kommen über Definitionen einiger Begriffe und Versuche nicht hinaus.

Die Pflanze besteht, abgesehen vom Wasser, zum gröBeren Teil aus Zellulose, ferner aus Lignin ${ }^{3}$ ), untergeordnet aus Ilarzen, Wachs und Fetten. Der Reichtum an Zellulose verleitet zu dem SchluB, daß sie hauptsächlich das Urmaterial der Humuskohle ist, deren vegetabilischer Ursprung, und zwar zumeist aus einer Sumpfvegetation, heute keinem ernsthaften Widerspruch begegnen dürfte.

Die Vertorfung ist als ZersetzungsprozeB das Mittelglied zwischen Vermoderung und Fäulnis, wobei zuerst die Vermoderung bei ungenügendem Luftzutritt, dann die Fäulnis im Wasser bei vollständigem Sauerstoffabschluß eintritt. Bei Vermoderung (Holzzerfall) ergibt sioh aus den Versuchszahlen von Rose und Lisse das Bild Fig. 1. Der Gehalt an Zellulose verarmt sehr rasch, während jener der alkalilöslichen Huminsäuren fast im gleichen MaBe ansteigt, ein Beweis dafür, daß diese aus jener gebildet wurden. Die Huminsâuren töteten die Bakterien ${ }^{5}$, deren Tätigkeit von manchen Forschern somit ganz bedeutend überschätzt wurde. Im Torf ist die Pflanzenstruktur lange erhalten, es kann also die Zellulose durch Bakterien nicht so rasch und vollständig gelöst werden, wie dieg Fischer-Schrader voraussetzen. Die Methoxylgruppe $\left(\mathrm{OCH}_{3}\right)$, welche das Lignin kennzeichnet, hat sich verdoppelt, kann also nicht die Huminsäuren geliefert haben, da sie in diesem Falle mehr oder weniger abgebaut worden wäre; ist der Methoxylgehalt des Holzlignins $15 \%$, so ist der Ligningehalt im ganz vermoderten Holz auf $30 \%$ gestiegen. Das Lignin ist also gegen Vermoderung ganz bedeutend widerstandsfähiger als die Zellulose. Nach dieser 1., der Zellulosephase (Vermoderung) mit reicher Huminsäurebildung

3) Die chemische Struktar der Lignine, eines Kolloides, ist noch fraglich, ob aliphatisch oder aromatisch; $E$. Bakmann u. O, Liesche unterscheiden $\alpha$-Lignin $\left(\mathrm{C}_{22} \mathrm{H}_{29} \mathrm{O}_{7}=405\right)$ und $\beta$-Lignin $\left(\mathrm{C}_{18} \mathrm{H}_{18} \mathrm{O}_{8}\right.$ $=390)$.

4) Journ. of Ind. and Engg. Chemistry 9, 284, 1917, durch Fischer-Sehrader. - Die Zahlen zu den 3 Diagrammen s. $F$. Fischer, Die Naturwissenschaften, Heft $47,1921$.

5) Die Mitwirkung der Bakterien beim Koblungsprozeß bezweifle ich ans denselben Grinden wie Dan nenberg, Geologie der Steinkohlenlager I, S. 11. 
tritt eine 2. Phase der Vertorfung ein, in welcher das Lignin und die Huminsäuren nebst Bitumen fast ausschlieBlich der Gegenstand der Metamorphose sind, da der kleine Zelluloserest - etwa $1 / 7$, des ursprünglichen Gewichts - sich voraussichtlich rasch in Huminsäure verwandeln wird.

Friedrich ${ }^{6}$ ) untersuchte, bei $F$. Fischer den Velener Torf in verschiedenen Stadien bzw. Tiefen von $0.0,0,9$ und $1,8 \mathrm{~m}$, welche Zahlen in Fig. 2 zeichnerisch dargestellt sind; auch hier entspricht dem Fortschreiten der Vermoderung ein Ansteigen der Humussäuren, und es darf aus Analogie mit Fig. 1 ein Zerfall der Zellulose vorausgesetzt werden. Der geringe Gehalt an Methoxyl scheint darauf zu verweisen, daß der Torf reich an Sphagnum ist, welches

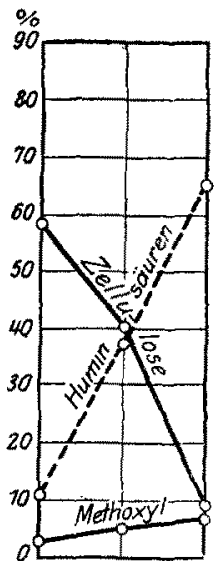

Fig. 1.

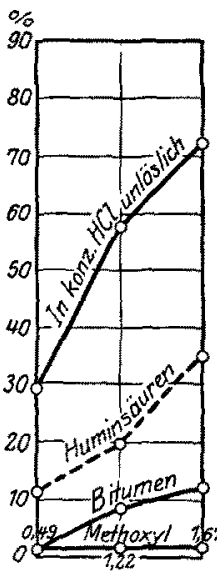

Fig. 2.

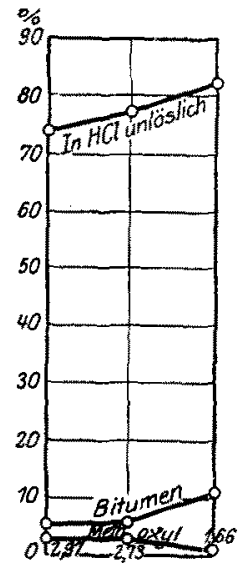

Fig. 3. Vermoderung und Vertorfung.

fast frei von Methoxyl ist. Der Gehalt an diesem bzw, an Lignin hat in $1,8 \mathrm{~m}$ Tiefe ebenso wie jener an Huminsäure um das 3,4 fache $z u$ genommen.

Die beiden Fig. 1 und 2 zeigen manche Ähnlichkeiten; da wie dort ist die fortschreitende Umwandlung durch ein stetiges Steigen der Huminsäuren und der Methoxylgruppe gekennzeichnet. Doch ist auffallenderweise der Gehalt an Huminsäure nicht auch beiderseits gleich, bei der Vermoderung $38,3 \%$, bei der Vertorfung nur $20 \%$. Von $0,9 \mathrm{~m}$ Tiefe steigt der Huminsäuregehalt im gleichen Maße wie er in der oberen Partie fortschreitet. Das untersuchte Velener Torflager ist bis $1.8 \mathrm{~m}$ Tiefe in der Zellulosephase, wobei der Gehalt an in konzentrierter IICl unlöslicher Substanz rasch zunimmt.

Friedrich ${ }^{B}$ untersuchte auch den älteren, schwarzen Torf von Lauchhammer (Provinz Sachsen) (Fig. 3). Der Gehalt an Methoxyl, also auch an Lignin, nimmt mit der Tiefe $a b$, ist in $3 \mathrm{~m} \mathrm{2,97,} \mathrm{in} 4 \mathrm{~m} \mathrm{2,73}$, in $5 \mathrm{~m}$ nur 1,66\%; innerhalb dieser Phase der Vertorfung setzt dex Abbau des Lignins entschieden ein; die Ligninphase (Vertorfen) hat begonnen. Leider gibt S. 21. Dureh Fischer-gehrader, Di* Entstehung usw.
Friedrich die Gehalte an Huminsäuren ${ }^{7}$ nicht an jener an Bitumen steigt von 5,3 über 6.3 anf $12,2 \%$. Das Bitumen, im siedenden Benzol vollständig löslich, ist also im Torfprozeß der resistenteste Bestandteil; es rührt vorwiegend von tierischen Resten, z. T. auch von dem Wachs und den Eiweißkörpern der Pflanzen her. Harzreiches Holz, z. B. Nadelholz, ist wegen des Harzgehaltes in der Vertorfung widerstandsfähiger als anderes. Aus den mitgeteilten Zahleri des Bitumengehaltes errechnet sich der Gewichtsverlust von der Tiefe 3 bis zu 5 mit $42.5 \%$, falls der Bitumengehalt in beiden Tiefen ursprïnglich gleich groß war.

Der Vertorfungs- oder Huminifizierungsprozeb ist als eine langsam verlaufende Anhydridisierung unter teilweiser Oxydation aufzufassen (Gr. Odén).

Die Huminsäuren, mehr aus Zellulose als aus Lignín entstanden. geben der Restmasse die braune Farbe; sie haben sich stetig angereichert, und eine warme Sodalösung wird deshalb intensiv braun gefärbt, um so weniger, je weiter die Verwandlung. d. i. nun der Kohlungsprozeß, vorgeschritten ist. Der Torf büBt bei weiter fortschreitender Umwandlung seine Struktur immer mehr ein und geht in das Braunhohlestadium über, innerhalb welehem sich der Rest der Hnminsäuren durch Oxydation und Wasseraustritt in das in Alkalien unlösliche Humin, nun der wesentliche Teil der Kohle, alimählich verwandelt; auch der Ligninrest wind ganz abgebant. Das Humin wird schon in diesem Stadium durch Oxydation, dem KohlungsprozeB, teilweise umgewandelt, karbonisiert. Sind diese Prozesse beendet, so ist die Braunkohle in das Schwarzkohlestadium eingetreten, die Umwandlung des zerstörten Humins setzt sich fort, die Alkalilösung wird nicht mehr braun gefärbt, und die Reaktion anf Tignin bzw. Methoxyl versagt. In Verlauf dieses Stadiums verliert die Koble Whren H- und O-Gehalt allmählich fast ganz und der Kohlenstoff hat sich im Anthrazit anf etwa $95 \%$ angereichert. Scheiden die beiden früher genannten Elemente aus, so entsteht der Graphit.

Wie bei diesem Tmwandlungsprozeli aus dem urspringlich nicht aromatisehen Material auch Stoffo mit aromatischen Figenschaften entstehen konnten, ist bisher fraglich.

Die Braunkohle besteht aus Wasser. Bitumen, Huminsänren. ans orarnischen in gewöhnlichen Tösungsmitteln nnlöslichen Substanzen, die $\mathrm{m}$. $\mathrm{E}$. vorwiegend Tumin sein dürften (Restkohle), und Asche. Deutsche Schwefellkohle enthält nach $E$. Erdmanne) (asche- wnd wasserfrei herechnet) $18.1 \%$ Bitumen, 49.3\% Huminsäuren und $38,36 \%$ Restkohle.

Bei dem besprorhenen Tmwandlungsprozeß

7) Der Gehalt an Huminsinren im Specktorf wird von anderer Seit mit $40-50 \%$ des Trockengewichtes angegeben, wahrscheinlich hat sich ein Teil der Huminsüuren durch Oxydation in Fumin umresetzt.

8) Kroy-Festschrift S. 309. 
der Holzsubstanz bis zur Schwarzkohle entwickelte sich Wärme; wie grol dieselbe im Torfstadium (Zellulose- und Ligninphase) ist, darüber liegen mir keine Beobachtungen vor. Aus den Hunderten genauer Messungen in den altösterreichischen Braunkohleflözen ${ }^{9}$ ) ermittelte ich für die Kohlungswärme, d. i. der WärmeüberschuB des Flözes gegenüber der seiner Tiefenlage entsprechenden Erdwärme, in NW. Böhmen für das westliche Revier mit bis $11,34^{\circ} \mathrm{C}$, im Ostrevier (Brüx-Teplitz) mit $16,35^{\circ}$ C. Die durch den Kohlungsprozeß entstandene Kohlungswärme nimmt mit der Tiefe, also auch mit der Flözwärme zu, u. zw. im Westrevier für je $100 \mathrm{~m}$ Tiefe um 5,61 ${ }^{\circ}$, im Ostrevier $11 \mathrm{~m}$ 1,28 . bis $19,8^{\circ}$ C. Im Schwarzkohlestadium pflegen die jüngsten oberkarbonen Flöze des OstrauKrakauer Steinkohlengebiets noch hier und da etwas Kohlungswärme zu besitzen, bei den tieferen jedoch scheint die Kohlung fast still zu stehen oder die erzeugte Wärme ist so gering, dab infolge der sie begleitenden stetigen $\mathrm{Ab}$ leitung ins Nebengestein nicht nachgewiesen werden kann. Für ungewöhnlich hohe Temperatur in der Gegenwart konnte ich in den österreichischen Kohlenbecken keine Beweise finden.

Verschiedene Geologen und Chemiker, von A. Petzholdt bis E. Donath, nehmen an, daß die Kohle bei ihrer Entwicklung einen flüssigen und breigen Zustand durchlaufen habe. Im Kohlestadium halte ich dies für ausgeschlossen, doch im Torfstadium für leicht möglich.

Dal bei dem Torfkohlungsprozeß dar Kohlenstoff sich durch allmähliches Ausscheiden von Wasser-, Sauer- und Stickstoff relativ anreichert, ist allbekannt; dabei entwickelt sich $\mathrm{CO}_{2}$, $\mathrm{H}_{2} \mathrm{O}$ und $\mathrm{CH}_{4}$. Das $\mathrm{CO}_{2}$ ist dic Folge der dunklen Verbrennung des $\mathrm{C}$ in dem $\mathrm{O}$ der Kohle, welche die Kohlungswärme bedingt und die Kohlung beschleunigt. Der Verbrauch an $O$ ist im Braunkohlestadium größer, wie dies die Analysen lehren, weshalb anch die Kohlungswärme gröBer als in der Schwarzkohle ist. Ber$\left.g_{i u s^{10}}\right)$ sagt auf Grund seiner fundamentalen Versuche, ,dab der Verkohlungsvorgang, d. h. der Zellulosezerfall, ein Wärme liefernder Prozell ist. Seine Wärmetönung ist sogar sehr grob. Sie beträgt ungefähr 70000 Cal. pro Mol". Das $\mathrm{CO}_{2}$ wird z. T. zur Zersetzung der unorganisehen Bestandteile (Asche), und zwar nicht bloß der Karbonate, sondern auch der Silikate, verwendet, die z. T. als wasserlösliche Bikarbonate entführt werden, woraus auch erklärlich ist, daß der Ascherehalt mit dem Fortschreiton der Kohlung nicht steigt.

Das $\mathrm{CH}_{4}$ kann auch dem Bitumen entstam-

9) II. v. Ihofer, Die geothermischen Verhältnisse der Kohlenbecken Üster reichs, Berg- u. Hüttenm. Jahrbuch, Wien 1917 .

10) Die Anwendung hoher Drucke bei chemischen Vorgïngen und eine Nachbildung des Entstehungs. prozerses der Steinkohle. S. 44, Halle a. S. 1912. men, welches, wie erwähnt, der Zersetzung am längsten widersteht und deshalb erst gegen Ende des Braunkohlestadiums und im Schwarzkohlestadium häufiger und reichlicher auftritt.

Es erübrigt noch jene Faktoren kennen zu lernen, welche den TorfkohlungsprozeB beschleunigten. Hier ist zuerst der Druck, der ja auch Wärme erzeugt, hervorzuheben. Es ist hier nicht so sehr der statische Druck des Hangenden als vielmehr der dynamische Druck der Dislokationen, besonders der Faltung makgebend. Das letztere habe ich bereits in meiner Arbeit: „Die geothermischen Verhältnisse der Kohlenbecken Österreichs" an geradezu schlagenden Beispielen aus Pennsylvanien (Karbon), aus verschiedenen Gebieten Steiermarks (Tertiär), aus Kreidekohlen Deutschlands und Osterreichs nachgewiesen. Hier kann auch die Verkohlung derHolzpiloten der Breisacher Rheinbrücke durch Stauchung erwähnt werden. Daß Wärme den Kohlungsprozeß befördert, ist allgemein bekannt, und zwar ist es 1, die Erdwärme, welche in dem MaBe steigt, wie das $\mathrm{Flöz}$ in gröbere Tiefe sinkt, 2. die Kohlungswärme, welche sich während des Kohlungsprozesses entwickelt, 3 . die dynamische Wärme, die während der dynamischen Veränderungen des Flözes frei wird Arbeit setzt sich in Wärme um. $\mathrm{Zu}$ diesen regionalen Wärmequellen kann 4. eine lokale treten, z. B. die Eruption von Gesteinen. Der statische Druck des Hangenden ist von geringem Einfluß.

Die Wirkung der Zeit an und für sich wird als geologische Potenz oft überschätzt; sie kann nicht abgestritten werden, da eingeleitete endogene chemische Prozesse länger Gelegenheit zum "Ausleben" haben; doch darf dabei nicht übersehen werden, daß ein Flöz, je älter es wird, meist mehr Episoden durch Druck und Wärme erlebte und deshalb stärker umgewandelt wurde. Ein krasses Beispiel von dem geringen Einfluß der Zeit ist das Kohlevorkommen im Unterkarbon (zwischen Devon und Bergkalk), also geologisch uralten, im Moskauer Becken ${ }^{11}$ ), welches Braunkohle führt, der man" sonst gewöhnlich nur in der jungen Tertiurformation begegnet. Die Flöze liegen fast wagerecht, das Hangende ist in der Yasenkigrube $60 \mathrm{~m}$ mächtig, der statische Druck scheint hier für den Kohlungsproze $B$ unbedeutend zu sein. Ich gebe drei Analysen dieser Kohle (wasser- und asohefrei berechnet) mit dem mindesten, mittleren und höchsten C-Gehalt, woraus hervorgeht, daß diese unterkarbone Kohle auch chemisch tatsächlich eine echte Braunkohle ist, worauf besonders auch der hohe Gehalt an $\mathrm{O}+\mathrm{N}$ verweist, die dunkle Verbrennung ist also relativ wenig fortgeschritten. $\mathrm{Ja}$, die erste Analyse entspricht dem Lignit und steht dem Torf näher als einer durchsehnittlichen Braunkohle. Der KohlungsprozeB ist also örtlich ver-

11) M. Prigorouski, The conl resources of the world ?. Bd, S. 1164 
schieden weit vorgeschritten, die Ursachen kann ich aus den mir zugänglichen Veröffentlichungen nicht ermitteln, vjelleicht liegt sie in den Mächtigkeiten des Deckgebirges.

\begin{tabular}{|c|c|c|c|}
\hline \multirow[t]{2}{*}{$\mathrm{Nr}$} & C & $\mathrm{H}$ & $0+N$ \\
\hline & \multicolumn{3}{|c|}{$0 \%$} \\
\hline 1 & $62,6 ?$ & 6,62 & 30,75 \\
\hline 2 & 72,69 & $\tilde{b}, 48$ & 21,93 \\
\hline 3 & 76,56 & 8,75 & 14,69 \\
\hline
\end{tabular}

Das ausgedehnte Moskauer Kohlenvorkommen verdient eine eingehende Untersuchung durch einen modernen Kohlengeojogen.

Die chemische Struktur der Kohle ist ausschlieBlich Sache der Chemiker; es stehen sich jetzt zwei Anschauungen gegenüber. Nach Marcusson u. a. enthält Huminsäure (sowie die aus ihr entstehende Braun- und Schwarzkohle) einen polymerisierten peri-Difuranring, ihr saurer Charakter ist auf Carboxylgruppen zurückzuführen. Fischer und Schrader behaupten dagegen, natürliche Huminsäure habe aromatische Struktur, zeige phenolartigen Charakter und enthalte keinen Furankern, da sie die Huminsäure rom Lignin ableiten.

\section{Zuschriften} und vorläufige Mitteilungen.

\section{Bemerkungen zu Koffkas Referat iiber}

W. Köhlers Arbeit: Die physischen Gestalten in Ruhe und im stationären Zustand.

Die von Kofflka in Hett 21 des 9. Jahrgangs dieser Zeitschrift zustimmend besprochene, im Titel zitierte Arbeit Köhlers bringt neben der Beurteilung physikalischer Zustunde, auf die jch nieht eingehen will, Anschaunngen iber Psychologisches, die nicht unwidersprochen bleiben dürfen. Nur in aller Kürze kann ich mich dazu äuBern.

Kohler sehliebt sich an Chr. v. Ehrenfels und Wertheimer an, welche in den phänomenalen Gestalten, in einer Melodie z. B., nicht bloB die summative Umgrenzung von Sinneseindrucken, bei der Melodie von Tönen, erblicken, sondern besondere dureh Ganzheit ausgezeichnete Gestaltqualitäten. Nach $v$. Ehrenfels handelt es sich dabei, im alten Sinne, um selustandige Leistungen des Nervensystems, Wertheimer aber ordnet die Gestalt direkt der Empfindung zu und Köhler tut nicht nur desgleichen, sondern findet sogar am Anorganen ganz entsprechende Erscheinungen, $z$. B. Eigenstrukturen elektrischer Leiter, die sich den lokalen Ladungsbetrïgen alis etwas Ganzes, Gestaltartiges, zugesellen sollen. Diesen Vergleich will ich hic $\mathrm{r}$ nicht diskutieren, da ich kein Physiker bin, wogegen ich aber Stellung nehmen muB, ist die innige Zuordnuag der Gestaltbildung, sagen wir: der Wahrnohmung, zar Empfindung.

Zunaichst ein Wort zur Neuronentheorie. dio von Köhler-Kofflo abgelehnt wird. Ohne sic bleibt uns der psychophysische Tatbestand unverständlich. Nur die Annahme selbständiger, mit spezifiechen Encrgien ansgestrteter Neurone garantiert die Selbstandigkeit rines beharrenden Empfindungsmosaiks, wie es im Rahmen jeder Wahrnehmung cegebon ist und in den
Erinnerungen unbegrenzt weiter lebt. Wirch dio Einzeleindrucke nicht in bestimmten Bahnen (Neurofibrillen) sozlusagen gefangen, so gäbe es im optischen Sektor nur einen diffusen Erregungsbrei, in dem wohl allerhand ,Eigenstrukturen" anftreten könnten, nber sicher keine phänomenalen Gestalten.

Und für die Wahrnehmung selbst bedarf es besonderer Neurone, Die moderne Tierpsychologie, die weder Köller noch Foffka wirdigen, hat mit Eviden dargetan, daß in Nervensystem die Form als Anlage parat liegt und sich nur mit jenen Reizkombinationen (Empfindungen) verbindet, welche ihr gestaltlich ent. sprechen. Das isi die Lehre von der Gegenwelt, welche v. Uexkiull und zur Straßen rein physiologisch, ich psychophysisch, ansgebaut haben. Nach ihr reagicren die Tiere nur dann auf Findricke, wenn sie diese mit angeborenen Konfigurationen, bzw. Gestaltdispositionen, im Nervensystem zur Deckung zu bringen vermögen. Dafür gibt es bereit eine Menge Beweise, uber die - noch unvollständig - mein 1912 erschienenes tierpsychologisches Praktikum orientiert; alls ihnen folgt aber die Selbstandigkeit, der Formpronesse von selbst.

Aber noch woitere Beweise gibt es für diese Solb. stitindigkeit. Auch bei uns Menschen erweist sich hutig genug die rambliche Walunchmung als ein von den Reizperzeptionen deutlich geschiedener Akt. Wir sind zweifellas gestaltlich viel reicher veranlagt als die Tiere, aber gar oft gleichen wir doch zuerst sehr der photographischen Platte, die nichts als ein Fleckenmosaik rezipiert, und erst in oft lange andauernder Arbeit vermögen wir dieses gestaltlich zu durchglie dern. Ich erinnere hier an das Studium mikroskopiseher Bilder, aber anch in der Natur passiort os uns oft genug, daß wir, vor allem in Minsicht auf die Raumtiefe, mühsam Formverstindnis uns crringen. Bei alledem tun wir im besonderen Falle nur das was ganz allomein das Kind in jahrelanger Entwicklungsarbeit, der sehent gwrordene Blindgeborene moichfalls nur allmällich leistet.

Tch finde, daB man der Ehrenfelsschen Gostalt. qualität überhaupt nicht gerecht wird, wenn man sie nur als ein Plus zur Summe der Empfintungen benrteilt, nicht als etwas vor aller Perzeption bereits Gegebenes, das in dicser nur realisiert, nicht geschaffen wirl. Eine Melodicgestalt zoigt das dentlichst, denn sie ist bei allen Einzeltönen der Melodie - die doch auch in der Erinnerung nur sukzessiv ins Bewubtsein treten - totaliter gegeben, ist daher otwas Apriorisches. Hier sei darauf verwiesen, daß nach Meinong, dem b kannten Grazer Gegenstandstheoretiker, Melodien, uberhaipt Gestaiten, nicht vom Kïnstler geschaffen, sondern nur entreckt, fl. h. ans latenter Anwesenheit im BewuBtsein erwekt und erst sekundïr za Tönen und anderem Material in Beziehnng gebracht werden; mir scheint aber, dag Ehrenfels gan? der gleichen, von Plato iibernommenen Auffassung iat.

Zum Schlusse möchte ich noch auf die aufallende Parallele des phänomeilologischen Gestaltoprozesses zur morphologischen Fintwicklung hinweisen. Wie die Entfaltung des Gestaltlichen am Körner abbängig ist von den Chromosomen, also der im Kern zegebenen Erbsubstanz, so auch die Wahrnehmung von bosonderen Nervenzellen, die wir direkt als gestaltbildende bezeichnen dürfen. SowohI die Entfaltung der Gewebe, die der Körpergestalt als Unterlage dienen, als auch die Empfindung, die gleiche Bedeutung hat für die Wahrnehmuns. verlïuft an sich selbstindig: die 